\title{
Role of Spinal Microglia in Visceral Hyperalgesia and NK1R Up-Regulation in a Rat Model of Chronic Stress
}

(Gastroenterology 2009;136:1339-1348)

Su Jin Hong, M.D.

Department of Internal Medicine, Soonchunhyang University School of Medicine, Bucheon, Gyeonggi-do, Korea

\section{Summary}

Chronic psychological stress is associated with visceral hyperalgesia. Microglia in the central nervous system are a type of nonneuronal cells and play a key role in the initiation or maintenance of hyperalgesia and allodynia, as demonstrated in animal models of chronic pain caused by peripheral inflammation or nerve injury. ${ }^{1}$ However, the mechanisms of microglia activation and the downstream pathways leading to amplification of the nociceptive signaling are only partially understood. Activated microglia produce and release a range of proinflammatory mediators, such as cytokines (IL-1, IL-6, tumor necrosis factor $\alpha$ ), chemokines, nitric oxide, and prostaglandins, all of which can act on afferent nerve terminals and spinal sensory neurons and increase neuronal excitability. ${ }^{2}$ The authors wanted to identify the role of spinal microglia in the development of visceral hyperalgesia from chronic stress in a rat model.

In the study, the authors used adult male Wistar rats with or without water avoidance (WA) stress. Rats were placed on a block $(8 \times 8 \times 10 \mathrm{~cm})$ affixed to the center of a Plexiglas cage $(25$ $\times 25 \times 45 \mathrm{~cm})$ filled with fresh water at room temperature $\left(25^{\circ} \mathrm{C}\right)$ to within $1 \mathrm{~cm}$ of the top of the block (WA) or kept empty (sham WA) for a period of one hour daily for 10 consecutive days. ${ }^{3}$ The rats had a surgically implanted chronic intrathecal (IT) catheter and an osmotic mini-pump. The rats then had a Teflon-coated electrode inserted into the external oblique musculature for electromyographic (EMG) recording. ${ }^{4}$

One group of rats was exposed to chronic WA stress or sham WA for 10 days and were sacrificed on day 11. Samples of lumbar spinal cord (L6S1) were collected and processed for immunohistochemically for P-p38, the microglia marker OX42, or the neuronal marker NeuN. Also, other groups of rats were exposed to WA stress or sham stress for 10 days and given daily injections of minocycline, the mitogen-activated protein kinase (MAPK) p38 inhibitor SB203580, or vehicle (0.9\% saline) via osmotic pump connected to an IT catheter. Phosphorylation levels of the kinase p38 (P-p38), the microglia marker OX42, $\mathrm{NK} 1 \mathrm{R}$, and $\mathrm{I} \kappa \mathrm{B} \alpha$ were assessed by immunoblotting and/or immunostaining of spinal samples. The authors also performed EMG recordings and baseline recordings of the visceromotor response (VMR) to colorectal distention (CRD) before beginning the treatment (day 0 ) and then again 24 hours after the last WA or sham WA sessions (day 11). The effects of the micro-

Received: September 28th, 2009 Accepted: November 2nd, 2009

(c) This is an Open Access article distributed under the terms of the Creative Commons Attribution Non-Commercial License (http://creativecommons. org/licenses/by-nc/3.0) which permits unrestricted non-commercial use, distribution, and reproduction in any medium, provided the original work is properly cited.

*Correspondence: Su Jin Hong, M.D.

Department of Internal Medicine, Soonchunhyang University School of Medicine, 1174 Jung-dong, Wonmi-ku, Bucheon, Gyeonggi-do 420-767, Korea

Financial support: None.

Tel: +82-32-621-5087, Fax: +82-32-621-5080, E-mail: sjhong@schbc.ac.kr

Conflicts of interest: None. 
glia-activating factor fractalkine were assessed on the visceral sensitivity in control nonstressed rats exposed to minocycline or vehicle.

The immunohistochemistry for the activation marker of microglia, P-p38, revealed greater P-p38 staining in the spinal dorsal horn from WA stressed rats compared with controls. The immunoreactivity for P-p38 colocalized with OX42-positive cells (microglia) from WA stressed rats. Western blotting also demonstrated increased levels of P-p38 in WA stressed rats compared with controls. This increase was inhibited by treatment with IT minocycline or with IT SB203580. Western blotting showed increased expression of NK1R in WA stressed rats compared with controls. Stress-induced increased NK1R expression was completely blocked by minocycline but not SB203580. WA stress induced decreased I $\mathrm{B} \alpha \alpha$ expression, and this decrease was partially blocked by minocycline and SB203580. WA stress induced hyperalgesia in stressed rats treated with vehicle. WA induced visceral hyperalgesia was blocked by daily treatment with IT injection of minocycline or SB203580. Fractalkine injection-induced hyperalgesia was blocked by minocycline.

\section{Comment}

Recurrent abdominal pain and discomfort without organic abnormalities is a principal symptom of functional gastrointestinal disorders such as irritable bowel syndrome (IBS). ${ }^{5}$ Previous studies have demonstrated that IBS patients exhibit lowered colorectal perceptual thresholds, increased sensory ratings, and larger viscerosomatic referral areas when compared with healthy individuals, consistent with heightened perception of visceral stimuli. ${ }^{6,7}$ Stress-induced visceral hyperalgesia is an important pathophysiological component of irritable bowel syndrome. ${ }^{8}$ The duration of stress or type and timing of the stressor might be a key factor in the determination of the visceral afferent pathways engaged, and will produce transient or sustained modulation of visceral nociception.

The mechanisms underlying chronic psychological stress-induced colonic hypersensitivity have been incompletely understood. Microglia are a type of glial cells in the spinal cord and the ambassadors to the CNS in the immune system. Some reports have recently shown the microglia responsiveness to environmental stressors without tissue injury in the rat brain. ${ }^{9,10}$ The spinal cord is also a candidate for neurological dysfunction in IBS. One study reported a potential role of spinal microglia in visceral hypersensitivity associated with neonatal colonic irritation in rats. ${ }^{11}$ However, the stress responsiveness of microglia in the spinal cord has not been studied before this article.

The authors used water avoidance, which has been proved to be a valid chronic psychological stressor in rodent models of sustained visceral hyperalgesia. ${ }^{3} \mathrm{WA}$ stress produced the increased level of P-p38 which is a marker of activated microglia in this rat model. Such activation might be developed by resident microglia rather than recruitment or proliferation of microglia because the level of spinal OX42 protein was not increased in WA stressed animals.

The increased expression of P-p38 in WA stressed rats was inhibited by treatment with IT minocycline, a potent inhibitor of microglia activation, ${ }^{12}$ or with IT the SB203580, the MAPK p38 inhibitor in this article. The reduction of the WA stress induced increase of $\mathrm{P}-\mathrm{p} 38$ by minocycline suggests that microglia are a key source of increased spinal P-p38 after chronic WA stress. Although the mechanism of minocycline action is biochemically unclear, it has been reported that inhibition of p38 MAPK activation may play a key role. ${ }^{13}$ The p38 MAPK inhibitor SB203580 could block WA stress-induced p38 phosphorylation and WA stress-induced visceral hyperalgesia. This finding supports that functional activation of spinal microglia affects stress-induced visceral pain.

On the other hand, chronic WA stress led to increased expression of NK1R in dorsal horn neurons. ${ }^{14}$ The authors observed that increased NK1R expression by WA stress was only blocked by minocycline in this experimental study. IL-1 $\beta$ or IL-6 affected NK1R gene expression in a prior report. ${ }^{15}$ This finding suggests that cytokines released from activated microglia during chronic WA stress were responsible for spinal NK1R up-regulation. SB203580 blocked WA stress-induced visceral hyperalgesia but did not affect NK1R up-regulation challenges. These findings suggest that there are multiple mechanisms participating in the development and maintenance of WA stress-induced visceral hyperalgesia, including spinal microglia activation and up-regulation of spinal NK1R.

In this study, the authors observed the activation of spinal microglia without proliferation and migration. Microglial migration has been demonstrated in the other pathologic lesions such as stroke, infectious disease, or neurodegenerative processes. Therefore, the exact functions of spinal microglia after WA stress may be explored in future studies. Activated microglia are also assumed to lead to increased NK1R expression which is not related to p38MAPK. Further study is required to show the pathways for visceral hyperalgesia via increased NK1R expression. And the 
roles of proinflammatory mediators such as IL-1 $\beta$, IL-6, and TNF- $\alpha$ should be assessed in this model for WA induced visceral hyperalgesia because this article could not explain the relationship between mediators and NK1R expression. In conclusion, this study shows new evidence for the role of spinal microglia activation in the development of visceral hyperalgesia in a model of chronic psychological stress in rats associated with up-regulation of the spinal NK1R system. This article enhances our understanding of chronic psychological stress-induced visceral hyperalgesia.

\section{References}

1. Schafers M, Sorkin LS, Geis C, et al. Spinal nerve ligation induces transient upregulation of tumor necrosis factor receptors 1 and 2 in injured and adjacent uninjured dorsal root ganglia in the rat. Neurosci Lett 2003;347:179-182.

2. Wieseler-Frank J, Maier SF, Watkins LR. Glial activation and pathological pain. Neurochem Int 2004;45:389-395.

3. Bradesi S, Schwetz I, Ennes HS, et al. Repeated exposure to water avoidance stress in rats: A new model for sustained visceral hyperalgesia. Am J Physiol Gastrointest Liver Physiol 2005; 289:G42-G53.

4. Ness TJ, Gebhart GF. Colorectal distension as a noxious visceral stimulus: physiological and pharmacological characterization of pseudoaffective reflexes in the rat. Brain Res 1988; 450:153-169.

5. Thompson WG, Longstreth GF, Drossman DA, Heaton KW, Irvine EJ, Mueller-Lissner SA. C. Functional bowel disorders and D. Functional abdominal pain. In: Drossman DA, Talley NJ, Thompson WG, Whitehead WE, Corazziari E, eds. Rome II: functional gastrointestinal disorders: diagnosis, pathophysiology, and treatment. 2nd ed. McLean: Degnon Associates Inc., 2000:351-432.

6. Bouin M, Plourde V, Boivin M, et al. Rectal distension testing in patients with irritable bowel syndrome: sensitivity, specificity, and predictive values of pain sensory thresholds. Gastroenterology 2002;122:1771-1777.

7. Mertz H, Naliboff B, Munakata J, Niazi N, Mayer EA. Altered rectal perception is a biological marker of patients with irritable bowel syndrome. Gastroenterology 1995;109:40-52.

8. Mayer EA, Naliboff BD, Chang L, Coutinho SV. Stress and the Gastrointestinal Tract V. Stress and irritable bowel syndrome. Am J Physiol Gastrointest Liver Physiol 2001;280:G519-G524.

9. Nguyen KT, Deak T, Owens SM, et al. Exposure to acute stress induces brain interleukin-1 $\beta$ protein in the rat. $\mathrm{J}$ Neurosci 1998;18:2239-2246.

10. Minami M, Kuraishi $Y$, Yamaguchi T, et al. Immobilization stress induces interleukin-1 beta mRNA in the rat hypothalamus. Neurosci Lett 1991;123:254-256.

11. Saab CY, Wang J, Gu C, Garner KN, Al-Chaer ED. Microglia: a newly discovered role in visceral hypersensitivity? Neuron Glia Biol 2007;2:271-277.

12. Zemke D, Majid A. The potential of minocycline for neuroprotection in human neurologic disease. Clin Neuropharmacol 2004;27:293-298.

13. Cui Y, Liao XX, Liu W, et al. A novel role of minocycline: attenuating morphine antinociceptive tolerance by inhibition of p38 MAPK in the activated spinal microglia. Brain Behav Immun 2008;22: 114-123.

14. Bradesi S, Kokkotou E, Simeonidis S, et al. The role of neurokinin 1 receptors in the maintenance of visceral hyperalgesia induced by repeated stress in rats. Gastroenterology 2006;130:1729-1742.

15. Simeonidis S, Castagliuolo I, Pan A, et al. Regulation of the NK-1 receptor gene expression in human macrophage cells via an NF-kappa B site on its promoter. Proc Natl Acad Sci USA 2003;100: 2957-2962. 